University of New Hampshire

University of New Hampshire Scholars' Repository

Space Science Center

Institute for the Study of Earth, Oceans, and

Space (EOS)

2001

\title{
COMPTEL observations of the Virgo blazars 3C 273 and 3C 279
}

W Collmar

Max-Planck-Institut für extraterrestriche Physik

V Schonfelder

Max-Planck-Institut für extraterrestrische Physik

S Zhang

Max-Planck-Institut für extraterrestriche Physik

H Bloemen

Space Research Organization of the Netherlands

W Hermsen

Space Research Organization of the Netherlands (SRON)

See next page for additional authors

Follow this and additional works at: https://scholars.unh.edu/ssc

Part of the Astrophysics and Astronomy Commons

\section{Recommended Citation}

COMPTEL observations of the Virgo blazars 3C 273 and 3C 279 Collmar, W. and Schönfelder, V. and Zhang, S. and Bloemen, $\mathrm{H}$. and Hermsen, W. and McConnell, M. and Bennett, K. and Williams, O. R., AIP Conference Proceedings, 587, 271-275 (2001), DOl:http://dx.doi.org/10.1063/1.1419412

This Conference Proceeding is brought to you for free and open access by the Institute for the Study of Earth, Oceans, and Space (EOS) at University of New Hampshire Scholars' Repository. It has been accepted for inclusion in Space Science Center by an authorized administrator of University of New Hampshire Scholars' Repository. For more information, please contact Scholarly.Communication@unh.edu. 
Authors

W Collmar, V Schonfelder, S Zhang, H Bloemen, W Hermsen, Mark L. McConnell, K Bennett, and O R. Williams 


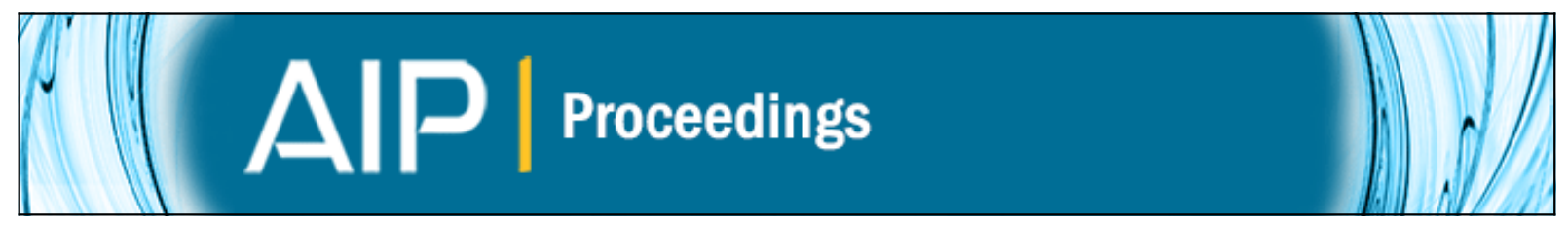

\section{COMPTEL observations of the Virgo blazars 3C 273 and 3C 279}

W. Collmar, V. Schönfelder, S. Zhang, H. Bloemen, W. Hermsen, M. McConnell, K. Bennett, and O. R. Williams

Citation: AIP Conference Proceedings 587, 271 (2001); doi: 10.1063/1.1419412

View online: http://dx.doi.org/10.1063/1.1419412

View Table of Contents: http://scitation.aip.org/content/aip/proceeding/aipcp/587?ver=pdfcov

Published by the AIP Publishing

Articles you may be interested in

Blazar surveys with WMAP and Swift

AIP Conf. Proc. 921, 32 (2007); 10.1063/1.2757262

Optical photometric observations of $y$-ray loud blazars in the October of 2000

AIP Conf. Proc. 587, 348 (2001); 10.1063/1.1419428

COMPTEL observations of the blazars 3C 454.3 and CTA 102

AIP Conf. Proc. 587, 343 (2001); 10.1063/1.1419427

Multifrequency observations of the Virgo blazars 3C 273 and 3C 279 in CGRO cycle 8

AIP Conf. Proc. 510, 303 (2000); 10.1063/1.1303222

Gamma-ray blazars

AIP Conf. Proc. 410, 307 (1997); 10.1063/1.54033 


\title{
COMPTEL Observations of the Virgo Blazars 3C 273 and 3C 279
}

\author{
W. Collmar ${ }^{1}$, V. Schönfelder ${ }^{1}$, S. Zhang ${ }^{1,5}$, H. Bloemen ${ }^{2}$, \\ W. Hermsen ${ }^{2}$, M. McConnell ${ }^{3}$, K. Bennett ${ }^{4}$, O.R. Williams ${ }^{4}$ \\ ${ }^{1}$ Max-Planck-Institut für extraterrestrische Physik, Garching, Germany \\ ${ }^{2}$ Space Research Organization Netherlands, Utrecht, The Netherlands \\ ${ }^{3}$ Space Science Center, University of New Hampshire, Durham, USA \\ ${ }^{4}$ Astrophysics Division, ESTEC, Noordwijk, The Netherlands \\ ${ }^{5}$ High Energy Astrophysics Lab, IHEP, P.O.Box 918-3, Beijing, China
}

\begin{abstract}
We report the main $\mathrm{MeV}$ properties (detections, light curves, spectra) of the Virgo blazars 3C 273 and 3C 279 which were derived from a consistent analysis of all COMPTEL Virgo observations between 1991 and 1997.
\end{abstract}

\section{INTRODUCTION}

The Virgo blazars 3C 273 and 3C 279 are well-known flat-spectrum radio quasars. Both sources have been detected at $\gamma$-ray energies by different instruments aboard the Compton Gamma-Ray Observatory (CGRO) from $~ 50 \mathrm{keV}$ (OSSE) up to 10$20 \mathrm{GeV}$ (EGRET). In this paper we summarize their main MeV properties, which are derived from a consistent analysis of all COMPTEL observations between 1991 and 1997. A complete description of the analysis results, put into multifrequency perspective, is in preparation [1].

\section{OBSERVATIONS AND DATA ANALYSIS}

The imaging Compton Telescope COMPTEL - one of four experiments aboard CGRO - was sensitive to $\gamma$-rays in the energy range $0.75-30 \mathrm{MeV}$ (for more details on COMPTEL see [2]). During 1991 and 1997 (i.e., CGRO observational phases I to VI) both sources were many times within the COMPTEL field-of-view (see Table 1). These data have been analysed - as a whole as well as individually - by using the standard COMPTEL maximum-likelihood analysis procedure including a filtering technique for background generation. Point spread functions assuming an $\mathrm{E}^{-2}$ power law shape for the source spectra were applied in our analyses.

CP587, GAMMA 2001: Gamma-Ray Astrophysics 2001, edited by S. Ritz et al. (C) 2001 American Institute of Physics 0-7354-0027-X/01/\$18.00 

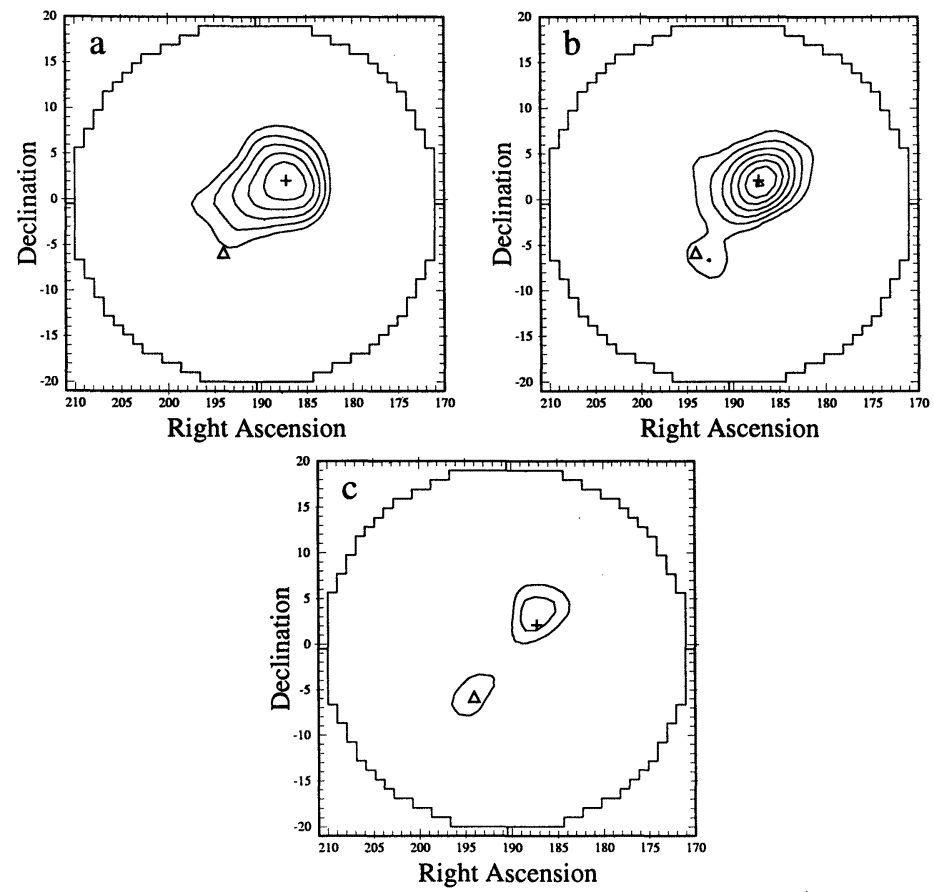

FIGURE 1. Time-averaged COMPTEL maximum-likelihood significance skymaps (1991 to 1997) of the Virgo region in 3 different bands (a: $1-3 \mathrm{MeV}$; b: $3-10 \mathrm{MeV}$; c: $10-30 \mathrm{MeV}$ ). The locations of the Virgo quasars 3C $273(+)$ and 3C $279(\Delta)$ are given. The contour lines for all maps start at $4 \sigma$ with a step of $1 \sigma$ ( $\chi_{1}^{2}$-statistics for a known source).

TABLE 1. COMPTEL exposures on 3C 273 and 3C 279 during CGRO Phases I to VI (1991 - 1997). The time periods of the different phases, the total observation times (days within COMPTEL field-of-view), and the effective COMPTEL exposures of $3 \mathrm{C} 273$ and $3 \mathrm{C} 279$ are given.

\begin{tabular}{lcccc}
$\begin{array}{l}\text { CGRO } \\
\text { Phase }\end{array}$ & Time Period & $\begin{array}{c}\text { Total } \\
\text { Obs. Time } \\
\text { [days] }\end{array}$ & $\begin{array}{c}\text { 3C 273 } \\
\text { Eff. Exp. } \\
\text { [days] }\end{array}$ & $\begin{array}{c}\text { 3C 279 } \\
\text { Eff. Exp. } \\
\text { [days] }\end{array}$ \\
\hline I & $05 / 91-11 / 92$ & 27 & 8.95 & 10.12 \\
II & $11 / 92-08 / 93$ & 21 & 5.58 & 5.51 \\
III & $08 / 93-10 / 94$ & 64 & 10.85 & 13.90 \\
IV & $10 / 94-10 / 95$ & 42 & 11.46 & 9.64 \\
V & $10 / 95-10 / 96$ & 14 & 6.42 & 6.21 \\
VI & $10 / 96-11 / 97$ & 49 & 15.08 & 12.07 \\
\hline Sum & $05 / 91-11 / 97$ & 217 & 58.3 & 57.5 \\
\hline
\end{tabular}




\section{RESULTS}

\section{$3 \mathrm{C} 273$}

In the sum of the 1991 - 1997 data (Table 1), 3C 273 is significantly detected in the 3 standard COMPTEL bands above $1 \mathrm{MeV}$. The detection significances are $\sim 9 \sigma$ in the $1-3 \mathrm{MeV}, \sim 9.5 \sigma$ in the $3-10 \mathrm{MeV}, \sim 6 \sigma$ in the $10-30 \mathrm{MeV}$ band (Fig. 1), which make 3C 273 the most significant $(\sim 15 \sigma, 1-30 \mathrm{MeV})$ COMPTEL AGN overall. Evidence for the source is found in about $80 \%$ of the individual Virgo pointings (Fig. 2). Within uncertainties, no time variability is seen in each of the 3 bands on timescales of a few weeks.

The time-averaged COMPTEL MeV spectrum for the sum of the observations between 1991 and 1997 (Table 1) is shown in Fig. 3 in two representations. The left panel gives the spectrum in the 4 standard COMPTEL bands. The fluxes in these bands indicate a curved time-averaged $\mathrm{MeV}$ spectrum of $3 \mathrm{C} 273$, which changes from a soft shape at upper COMPTEL energies to a harder one at lower energies. The emission maximum, and therefore the peak of the nonthermal inverse-Compton radiation of 3C 273 (for a multifrequency spectrum see e.g. [3]), is located in the 3$10 \mathrm{MeV}$ band. A power-law fit to this summed spectrum results in an unacceptable $\chi^{2}$-value of $\sim 13$ for 2 degrees of freedom, proving that a power law is not a viable model. The right panel shows a preliminiary 'high-resolution' (11 data points) spectrum of the same data which could be obtained because of the relatively high statistics for 3C 273. Again a spectral turnover at $\mathrm{MeV}$ energies is evident. This spectrum suggests that the spectral turnover of $3 \mathrm{C} 273$ is on average broad and rather smooth. No spectral features (e.g., hints for a blueshifted annihilation line) are obvious.

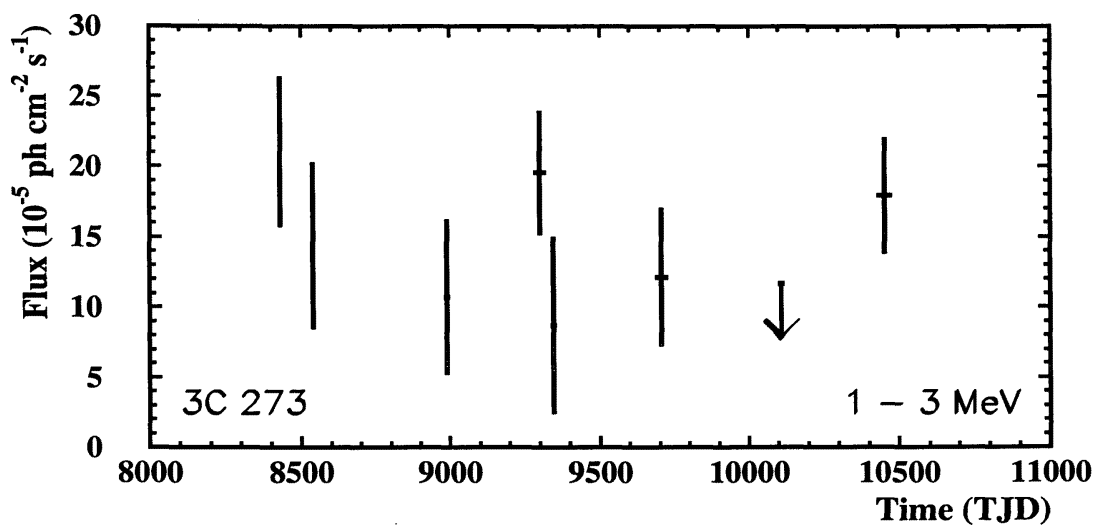

FIGURE 2. COMPTEL light-curves of $3 \mathrm{C} 273$ in the $1-3 \mathrm{MeV}$ band along the CGRO mission between 1991 and 1997. The blazar shows a rather stable $\gamma$-ray emission. The errors are $1 \sigma$ and the upper limit is $2 \sigma$. 

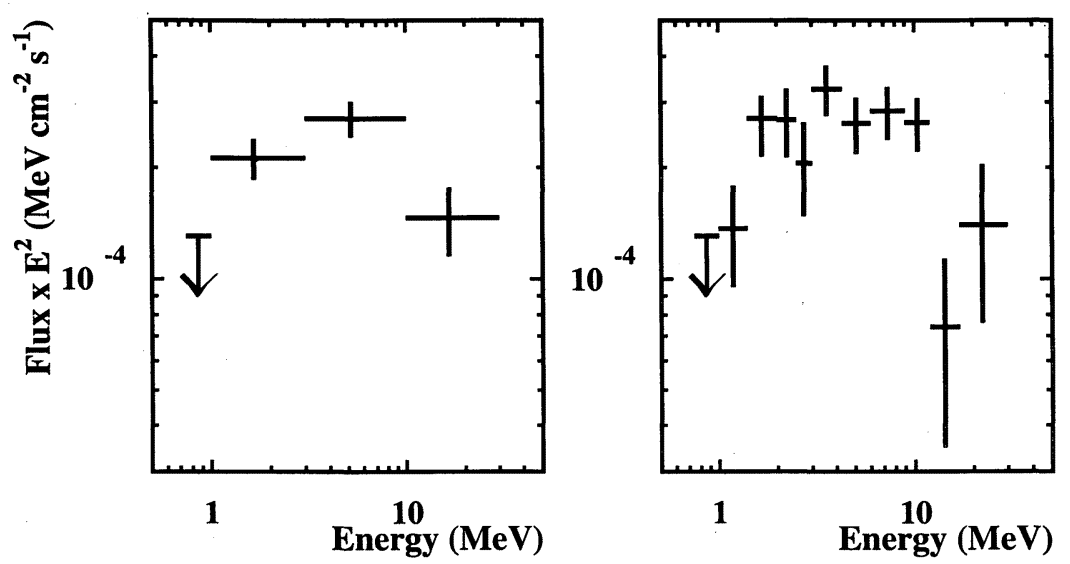

FIGURE 3. Time-averaged (i.e., for the sum of the observations in Table 1) COMPTEL MeV spectra of $3 \mathrm{C} 273$. The left panel shows the spectrum in the 4 standard COMPTEL bands (0.75-1, 1-3, 3-10, 10-30 MeV), and the right one shows the same spectrum with increased spectral resolution (11 spectral points). The errors are $1 \sigma$ and the upper limits are $2 \sigma$.

\section{$3 \mathrm{C} 279$}

In the sum of all 1991 - 1997 data there is evidence for 3C 279 at energies above $\sim 1 \mathrm{MeV}$, more significantly above $\sim 3 \mathrm{MeV}$ (Fig. 1). The detection significances for this period are $\sim 3.5 \sigma, \sim 4.5 \sigma$, and $\sim 4.5 \sigma$ in the $1-3 \mathrm{MeV}, 3-10 \mathrm{MeV}$, and $10-$ $30 \mathrm{MeV}$ COMPTEL bands, respectively. The detections and non-detections along the course of the mission indicate time variability (Fig. 4). This is most obvious by the high flux in the 10-30 MeV band during CGRO VP 511.5 (February '96), which is about $3 \sigma$ above the previous flux measurement and the upper limit in the next observational period, and which is simultaneous to the largest $\gamma$-ray flare ever observed from 3C 279 by EGRET. There is the trend, that 3C 279 becomes visible in the uppermost COMPTEL band, when EGRET observes a strong flaring event (Fig. 4).

The COMPTEL MeV spectra of 3C 279 can be well represented by power-law shapes. The 6-year time-averaged spectrum is consistent with a power-law shape $\left(\mathrm{E}^{-\alpha}\right)$ with a photon index $\alpha$ of $1.78 \pm 0.15$ (Fig. 5). The spectra for the individual time periods are less well determined, but they indicate the trend of a spectral hardening with EGRET-measured flux. To investigate this trend, we subdivided the COMPTEL data according to the $\gamma$-ray state (high vs. low) above $100 \mathrm{MeV}$, and generated $\mathrm{MeV}$ spectra for the sum of both states (Fig. 5). These two spectra show different spectral shapes with a crossover at $\sim 2 \mathrm{MeV}$. This indicates that the 'EGRET flares' are a high-energy $\gamma$-ray $(>3 \mathrm{MeV})$ phenomenon, and that the peak of the inverse-Compton emission changes with source flux in 3C 279. 


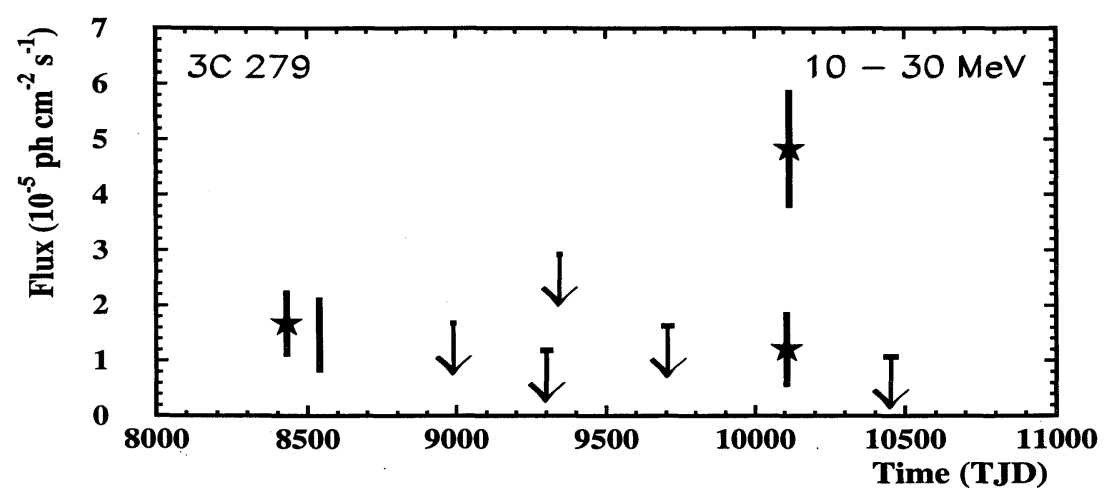

FIGURE 4. COMPTEL light curve of 3C 279 in the $10-30 \mathrm{MeV}$ band from 1991 to 1997. The detections and non-detections indicate time variability. The blazar is always detected by COMPTEL when EGRET reports $\gamma$-ray flaring (i.e, high fluxes) at energies above $100 \mathrm{MeV}$. These periods are marked by a ' $\star$ '.
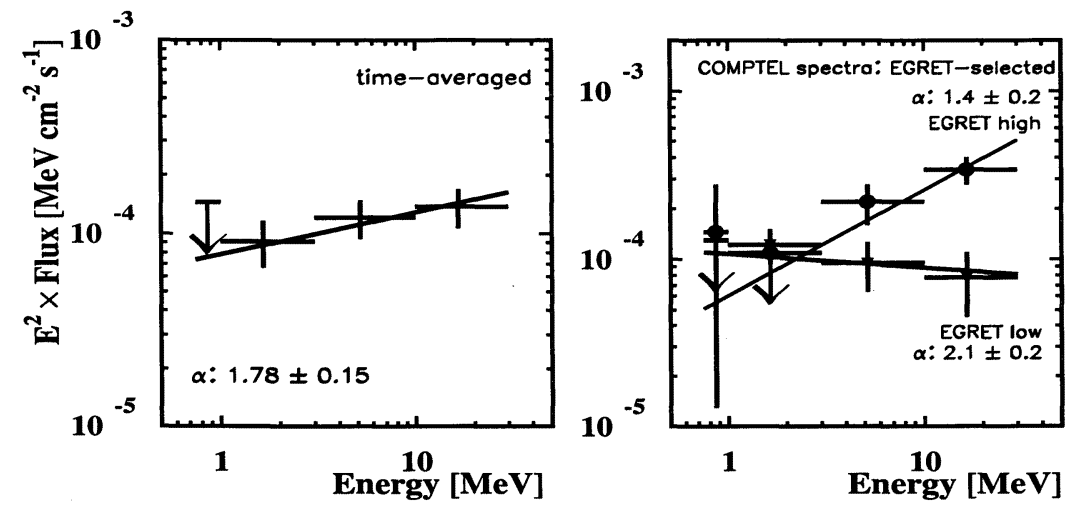

FIGURE 5. Time-averaged COMPTEL spectra of 3C 279. The left panel shows the spectrum averaged over the sum of the ' 91 to ' 97 data, while the right panel shows spectra averaged over times of $\gamma$-ray flaring ('EGRET high') and $\gamma$-ray quiescent ('EGRET low') periods. The spectral difference is obvious.

ACKNOWLEDGMENTS: The COMPTEL project is supported by the German government through DARA grant 50 QV 9096 8, by NASA under contract NAS5-26645, and by the Netherlands Organization for Scientific Research (NWO).

\section{REFERENCES}

1. Collmar, W., et al., in preparation (2002).

2. Schönfelder, V., et al., ApJS 86, 657 (1993).

3. Lichti, G., et al., $A \mathscr{E} A$ 298, 711 (1995). 